\title{
Plano de amostragem do biótipo B de Bemisia tabaci na cultura do pepino(1)
}

\author{
Marcelo Fialho de Moura(2), Marcelo Coutinho Picanço(2), Ézio Marques da Silva(2), \\ Raul Narciso Carvalho Guedes ${ }^{(2)}$ e Jardel Lopes Pereira ${ }^{(3)}$
}

\begin{abstract}
Resumo - A adoção de programas de manejo integrado de pragas permite reduzir a aplicação de inseticidas e os planos convencionais de amostragem representam o ponto inicial na geração desses programas. Assim, o objetivo deste trabalho foi determinar plano de amostragem convencional para o biótipo B da mosca-branca Bemisia tabaci Genn. (Hemiptera: Aleyrodidae) na cultura do pepino. Foram avaliadas as densidades de ninfas e de adultos em dez lavouras comerciais de pepino, Tocantins, MG, estudando nove sistemas amostrais formados pela combinação de três técnicas (batida de folha em bandeja de plástico branco, contagem direta dos insetos na face inferior da folha e coleta de folha em sacola de plástico) e três unidades amostrais (folha do terço apical, mediano ou basal do espaldeiramento). A contagem direta em folha do terço basal foi o sistema com maior precisão econômica na amostragem de ninfas, mas não possibilitou a geração de plano de amostragem praticável. As batidas, em bandeja, de folha dos terços apicais, medianos ou basais do espaldeiramento foram os sistemas economicamente mais precisos na amostragem de adultos. Desses, apenas a batida de folha do terço mediano em bandeja gerou plano de amostragem praticável, sendo que este plano é composto de 196 amostras/lavoura.
\end{abstract}

Termos para indexação: Cucumis sativus, mosca-branca, ninfa, manejo integrado de praga.

\section{Sampling plan for B-biotype of Bemisia tabaci in cucumber crop}

\begin{abstract}
The adoption of pest management integrated programs decreases insecticide applications, and standardized sampling plans represent the initial point in the generation of those programs. This work aimed to determine a conventional sampling plan for B-biotype of the whitefly Bemisia tabaci Genn. (Hemiptera: Aleyrodidae) in cucumber crops. The densities of nymphs and adults were evaluated in ten commercial crops in Tocantins, Minas Gerais State, Brazil. Nine sampling systems compared three sampling techniques, which were the beating of leaf on white plastic trays, the direct counting of insects on abaxial leaf surface, and the leaf collection in plastic bag, and three sample units, a leaf on the apical, median, and basal thirds of the training system. The system with the largest economic precision in the nymphs sampling was the direct counting on leaf of the basal third of the training system. However, that system did not generate a practicable sampling plan. The systems with the largest economic precisions in the adults' sampling were the beating on plastic trays of a leaf from the apical, medium or basal portions of the training system. Only the sampling system of beating leaves of the third medium on plastic trays generated a practicable plan. The number of samples for the adults' sampling on the leaves of the medium portion of the training system was of 196 per field.
\end{abstract}

Index terms: Cucumis sativus, white flies, nymphs, integrated pest management.

\section{Introdução}

De acordo com dados da FAO, o continente com maior produção de pepino, no ano de 2002, foi a Ásia

(1) Aceito para publicação em 23 de outubro de 2003.

(2) Universidade Federal de Viçosa (UFV), Dep. de Biologia Animal, Avenida P. H. Rolfs s/n, CEP 36571-000 Viçosa, MG. Bolsista do CNPq. E-mail: mfmoura@alunos.ufv.br, picanco@ufv.br,ezioms@insecta.ufv.br,guedes@mail.ufv.br

(3) UFV. Bolsista da Fapemig. E-mail: jardel.ufv@bol.com.br
(91,57\%), seguida pelas Américas $(5,64 \%$, sendo $5,27 \%, 0,14 \%$ e $0,24 \%$ nas Américas do Norte, Central e do Sul, respectivamente), África (1,54\%), Europa $(1,18 \%)$ e Oceania $(0,06 \%)$, sendo que nos dados da América do Sul não está relatada a produção brasileira. No Brasil, o único estado com registro, ao longo dos anos, da produção de pepino é Minas Gerais, em que se destacam os municípios de Mateus Lemes (15,63\%), Uberlândia (8,58\%), Baldim (8,58\%), Araguari $(8,56 \%)$ e Tocantins $(7,71 \%)$. 
A raça $\mathrm{B}$ da mosca-branca Bemisia tabaci (Genn.) (Hemiptera: Aleyrodidae) é responsável por grandes perdas na produtividade do pepino e de diversas outras hortaliças no Brasil (Silva et al., 1992; Filgueira, 2000) e em outros países (Brown, et al., 1995; Ellsworth \& Carrillo, 2001; Naranjo, 2001). Normalmente, os agricultores adotam o controle químico como única medida de controle desse inseto-praga. Porém, o uso inadequado do controle químico causa elevação do custo de produção e poluição do solo, ar e água (Picanço \& Guedes, 1999; Picanço et al., 2001). A adoção de programas de manejo integrado de pragas, no entanto, possibilita reduzir as aplicações de inseticidas (Barbosa \& Perecin, 1982).

Os planos convencionais de amostragem representam importante instrumento na tomada de decisão no controle de pragas (Pedigo, 1988), por configurarem o ponto inicial de geração de sistemas nos programas de manejo integrado de pragas. Permitem, ainda, a determinação do prejuízo econômico e a escolha da melhor unidade e técnica amostral, além de servir como padrão de validação dos planos seqüenciais (Nault \& Kennedy, 1996; Gusmão, 2000). Um plano de amostragem convencional é composto de sistema amostral e número fixo de amostras. O sistema de amostragem é constituído por técnica e unidade amostrais e indica como e onde será realizada a amostragem. Já o número de amostras deve ser tal que possibilite uma amostragem precisa, rápida e de baixo custo (Bliss \& Owens, 1958; Pedigo et al., 1982; Pedigo, 1988; Gusmão, 2000).

A batida de folhas em bandeja de fundo branco, a contagem direta dos insetos nas folhas (Paula et al., 1997; Miranda et al., 1998; Gusmão, 2000), e a coleta de folhas em sacola de plástico (Waquil, 1997) são técnicas normalmente empregadas na avaliação de insetos da ordem Hemiptera, como a mosca-branca. Entretanto não se conhece a precisão destas técnicas na determinação das populações da mosca-branca em cultivos de pepino e a distribuição de probabilidade da praga, atividade de total relevância, pois possibilita selecionar técnicas adequadas de análises estatísticas dos dados de amostragem (Barbosa \& Perecin, 1982; Schuster, 1998).

O objetivo desse trabalho foi determinar um plano de amostragem convencional para ninfas e adultos do biótipo B de B. tabaci em cultivo de pepino.

\section{Material e Métodos}

O trabalho foi realizado de julho a dezembro de 2003 em Tocantins, MG, em dez lavouras comerciais de pepino da cultivar Caipira cujos frutos seriam destinados ao consumo in natura. Cada lavoura possuía cerca de 1 ha e foi plantada em espaçamento de $1 \mathrm{~m}$ entre linhas e $0,5 \mathrm{~m}$ entre plantas. Os tratos culturais foram realizados conforme Filgueira (2000) com utilização de mão-de-obra familiar, tração animal, irrigação por sulco e de adubos químicos.

Quando as plantas estavam em fase reprodutiva, monitoraram-se as densidades de ninfas e de adultos do biótipo B de B. tabaci. As plantas avaliadas localizavamse equidistantemente ao longo e entre as linhas de plantio, de modo a cobrir toda a área plantada e eliminar tendências direcionais (Midgarden et al., 1993).

Foram avaliadas as técnicas de batida de folha em bandeja de plástico branco $(35 \mathrm{~cm}$ de comprimento $\mathrm{x} 30 \mathrm{~cm}$ de largura $\times 5 \mathrm{~cm}$ de profundidade), a contagem direta dos insetos presentes na face inferior da folha e a coleta de folha em sacola de plástico para contagem em laboratório. A unidade de amostragem estudada foi uma folha de ramo localizado nos terços apical, mediano e basal do espaldeiramento (Paula et al., 1997; Miranda et al., 1998; Schuster, 1998), por ser o local de colonização da moscabranca (Schuster, 1998). As plantas avaliadas foram distintas em cada sistema de amostragem, de modo a evitar qualquer perturbação aos insetos. As ninfas foram avaliadas utilizando a técnica de contagem direta e a coleta de folha em sacola de plástico. Na amostragem de adultos, utilizaram-se as três técnicas de amostragem. Nas contagens, não foram considerados os insetos mortos.

A técnica da contagem direta consistiu em virar cuidadosamente a folha, segurando-a pela ponta ou pelo pecíolo, de forma a não afugentar os adultos e, em seguida, contar os insetos presentes na face abaxial. A técnica de batida de folha em bandeja consistiu em sacudir a folha no interior da bandeja, usando-se movimentos bruscos e vibratórios, e, a seguir, contar os insetos presentes no fundo da bandeja. A técnica de coleta de folha em sacola de plástico, consistiu em envolver a folha com a sacola de plástico, arrancá-la, lacrá-la no interior da sacola e encaminhá-la ao laboratório para determinação do número de insetos (Paula et al., 1997; Waquil, 1997; Gusmão, 2000). Foram calculadas as médias e os erros-padrões das densidades de ninfas e adultos para cada sistema amostral.

Registraram-se os tempos e calcularam-se os custos de cada sistema de amostragem. Além disso, calcularam-se as médias e erros-padrões das densidades para cada sistema de amostragem, os quais foram utilizados no cálculo da variância relativa, de acordo com Pedigo et al. (1982). 
A variância relativa mede a variabilidade dos dados amostrais, e os melhores sistemas são os que apresentam menores valores para esta característica.

Com os dados do custo de amostragem (Ca) e variância relativa (VR), calcularam-se as precisões econômicas (PE) dos sistemas de amostragem com a expressão de Kogan \& Herzog (1980):

$\mathrm{PE}=100 /(\mathrm{VR} \times \mathrm{Ca})$.

A precisão econômica indica o melhor sistema de amostragem com base na variabilidade e custo amostrais, e os melhores sistemas são aqueles com os maiores valores em relação a esta característica. Os valores de precisão econômica e variância relativa foram submetidos à análise de variância e as médias dos sistemas amostrais comparadas pelo teste de Scott-Knott a 5\% de probabilidade.

$\mathrm{O}$ ajuste dos dados dos sistemas mais precisos de amostragem de adultos e de ninfas de $B$. tabaci aos modelos de distribuições de freqüência binomial negativa, binomial positiva e Poisson foi realizado utilizando-se o teste de qui-quadrado a $\mathrm{p}=0,05$ (Young \& Young, 1998). Como os dados dos sistemas mais precisos para a amostragem de adultos e ninfas se ajustaram à distribuição binomial negativa na maioria dos talhões e não se ajustaram às distribuições binomial positiva e Poisson, testouse a existência do parâmetro de agregação comum dos dados das lavouras $\left(\mathrm{K}_{\text {comum }}\right)$, conforme Bliss \& Owens (1958).

No cálculo do número de amostras adotou-se a fórmula: $\mathrm{N}=\left(\mathrm{Z}_{\alpha / 2} / \mathrm{D}\right)(1 / \mathrm{m}+1 / \mathrm{K})$,

em que $\mathrm{K}$ é o parâmetro da distribuição binomial negativa (utilizou-se o $\mathrm{K}_{\text {comum }}$ quando sua existência foi confirmada), mé a média da população, D é o nível de precisão esperado (adotou-se D a 5\%, 10\%, 15\%, 20\% e 25\% de precisão) e $Z_{\mathrm{a} / 2}$ é o valor da distribuição normal padrão a um nível de confiança $(1-\alpha)$ (Young \& Young, 1998).

$\mathrm{Na}$ inexistência de ajuste de $\mathrm{K}_{\text {comum }}$, os números de amostras foram calculados utilizando-se os valores de $\mathrm{K}$, para cada lavoura, estimados pelo método dos momentos, conforme a equação:

$\mathrm{K}=\mathrm{m}^{2} /\left(\mathrm{S}^{2}-\mathrm{m}\right)$

em que $S^{2}$ é a variância dos dados e $m$ a média da população (Young \& Young, 1998). Selecionaram-se os sistemas amostrais cujo número de amostras necessárias a compor o plano amostral exigiu um tempo inferior a uma hora (Gusmão, 2000).

\section{Resultados e Discussão}

As maiores densidades de adultos da mosca-branca foram detectadas pela contagem direta em folha do terço apical do espaldeiramento (Tabela 1), em razão da migração de fêmeas para ovipositarem em folhas novas (Gusmão, 2000), encontradas em maior número nessa região. Também contribuíram para a detecção de maiores densidades de adultos pela contagem direta em folha do terço apical a baixa dispersão e o baixo número de adultos mortos, quando amostrados por esta técnica. Quando se utilizou a técnica de batida em bandeja branca, ocorreu dispersão de parte dos adultos antes da contagem. Quando foi usada a sacola de plástico, parte dos insetos não foi contada porque morreram e ficaram aderidos às paredes da sacola, em razão da condensação de água proveniente da transpiração da folha.

As maiores densidades de ninfas foram detectadas na contagem direta em folha do terço basal e no ensacolamento de folha dos terços mediano e basal do espaldeiramento (Tabela 1). As maiores densidades de ninfas nesses sistemas amostrais foram em decorrência da maior proporção de ninfas visíveis a olho nu (terceiro e quarto instar) nas folhas mais velhas (Lourenção \& Nagai, 1994; Naranjo \& Flint, 1994; Schuster, 1998), localizadas, em maior proporção, nas partes baixas do espaldeiramento. Além disso, as folhas mais velhas proporcionam maior tempo de exposição à colonização pela mosca-branca (Gusmão, 2000).

Ao se considerar a variância relativa, todos os sistemas estudados foram adequados na amostragem de adultos de $B$. tabaci, por apresentarem valores inferiores a 25 (Tabela 2), limite utilizado na seleção de sistemas que devem fazer parte dos planos de

Tabela 1. Densidade (médiaterro-padrão) da mosca-branca Bemisia tabaci (Hemiptera: Aleyrodidae) em dez lavouras de pepino, em razão da técnica de amostragem e localização da folha no espaldeiramento. Tocantins, MG. 2001 ${ }^{(1)}$.

\begin{tabular}{|c|c|c|c|}
\hline \multirow[t]{2}{*}{ Técnica } & \multirow{2}{*}{$\begin{array}{c}\text { Parte do } \\
\text { espaldeiramento }\end{array}$} & \multicolumn{2}{|c|}{ Mosca-branca/amostra } \\
\hline & & Adultos & Ninfas \\
\hline \multirow[t]{3}{*}{ Batida em bandeja } & Apical & $3,32 \pm 0,92 \mathrm{~B}$ & $-(2)$ \\
\hline & Mediana & $2,33^{+} 1,02 \mathrm{C}$ & \\
\hline & Basal & $3,10^{ \pm} 1,92 \mathrm{~B}$ & - \\
\hline \multirow[t]{3}{*}{ Contagem direta } & Apical & $6,0{ }^{ \pm} 1,04 \mathrm{~A}$ & $2,11 \pm_{1,55 \mathrm{~B}}$ \\
\hline & Mediana & $3,04^{ \pm} 1,49 \mathrm{~B}$ & $6,03 \pm 3,70 \mathrm{~B}$ \\
\hline & Basal & $3,74^{+} 1,63 \mathrm{~B}$ & $17,69 \pm 8,39 \mathrm{~A}$ \\
\hline \multirow[t]{3}{*}{ Sacola de plástico } & Apical & $1,76^{ \pm} 0,76 \mathrm{C}$ & $5,88 \pm 3,44 \mathrm{~B}$ \\
\hline & Mediana & $1,60^{ \pm} 0,85 \mathrm{C}$ & $11,57 \pm 6,77 \mathrm{~A}$ \\
\hline & Basal & $1,66^{ \pm} 0.93 \mathrm{C}$ & $16,00 \pm 9,42 \mathrm{~A}$ \\
\hline
\end{tabular}

${ }^{(1}$ Médias seguidas pela mesma letra, em cada coluna, não diferem entre si pelo teste de Scott-Knott a 5\% de probabilidade. ${ }^{(2)}$ Técnica de amostragem não testada para ninfas. 
amostragem (Southwood, 1978; Gusmão, 2000). Utilizando-se esse mesmo critério, a contagem direta em folha do terço basal e o ensacolamento de folha dos terços mediano ou basal, foram considerados como sistemas adequados na amostragem de ninfas (Tabela 2). Os elevados valores de variância relativa, encontrados na amostragem de ninfas, com a conta-

Tabela 2. Variância relativa, tempo de amostragem, custo e precisão econômica das amostras de contagens de adultos e ninfas de Bemisia tabaci (Hemiptera: Aleyrodidae) por três técnicas de amostragem em folha do terço apical, mediano e basal do espaldeiramento de plantas de pepino. Tocantins, MG, 2001 ${ }^{(1)}$.

\begin{tabular}{|c|c|c|c|}
\hline Técnica & $\begin{array}{c}\text { Parte do } \\
\text { espaldeiramento }\end{array}$ & Adultos & Ninfas \\
\hline \multirow{4}{*}{ Batida em bandeja } & & \multicolumn{2}{|c|}{ Variância relativa (\%) } \\
\hline & Apical & $15,16 \mathrm{~A}$ & $-(2)$ \\
\hline & Mediana & $21,04 \mathrm{~A}$ & $-(2)$ \\
\hline & Basal & $11,89 \mathrm{~A}$ & $-(2)$ \\
\hline \multirow[t]{3}{*}{ Contagem direta } & Apical & $9,38 \mathrm{~A}$ & $39,88 \mathrm{~A}$ \\
\hline & Mediana & $15,10 \mathrm{~A}$ & $28,20 \mathrm{~A}$ \\
\hline & Basal & $15,72 \mathrm{~A}$ & $18,17 \mathrm{~B}$ \\
\hline \multirow[t]{3}{*}{ Sacola de plástico } & Apical & $20,92 \mathrm{~A}$ & $32,56 \mathrm{~A}$ \\
\hline & Mediana & $17,49 \mathrm{~A}$ & $20,03 \mathrm{~B}$ \\
\hline & Basal & $22.24 \mathrm{~A}$ & $19.79 \mathrm{~B}$ \\
\hline \multirow{4}{*}{ Batida em bandeja } & & \multicolumn{2}{|c|}{ Tempo (s/amostra) } \\
\hline & Apical & $16,79 \mathrm{C}$ & $-(2)$ \\
\hline & Mediana & $15,73 \mathrm{C}$ & $-(2)$ \\
\hline & Basal & $28,33 \mathrm{~B}$ & $-(2)$ \\
\hline \multirow[t]{2}{*}{ Contagem direta } & Apical & $30,26 \mathrm{~B}$ & \\
\hline & Mediana & $26,79 \mathrm{~B}$ & (3) \\
\hline \multirow{4}{*}{ Sacola de plástico } & Basal & $28,43 \mathrm{~B}$ & $51,12 \mathrm{~B}$ \\
\hline & Apical & $82,12 \mathrm{~A}$ & \\
\hline & Mediana & $81,90 \mathrm{~A}$ & $110,06 \mathrm{~A}$ \\
\hline & Basal & $82.35 \mathrm{~A}$ & $121.67 \mathrm{~A}$ \\
\hline \multirow{4}{*}{ Batida em bandeja } & & \multicolumn{2}{|c|}{ Custo (R\$/amostra) } \\
\hline & Apical & $0,015 \mathrm{C}$ & $\begin{array}{l}(2) \\
-(2)\end{array}$ \\
\hline & Mediana & $0,014 \mathrm{C}$ & $-(2)$ \\
\hline & Basal & $0,0252 \mathrm{~B}$ & $-(2)$ \\
\hline \multirow{2}{*}{ Contagem direta } & Apical & $0,0268 \mathrm{~B}$ & (3) \\
\hline & Mediana & $0,0238 \mathrm{~B}$ & (3) \\
\hline \multirow{4}{*}{ Sacola de plástico } & Basal & $0,0252 \mathrm{~B}$ & $0,0454 \mathrm{~B}$ \\
\hline & Apical & $0,0730 \mathrm{~A}$ & \\
\hline & Mediana & $0,0728 \mathrm{~A}$ & $0,0978 \mathrm{~A}$ \\
\hline & Basal & $0,0732 \mathrm{~A}$ & $0,1082 \mathrm{~A}$ \\
\hline \multirow{4}{*}{ Batida em bandeja } & & \multicolumn{2}{|c|}{ Precisão econômica } \\
\hline & Apical & $1.144,88 \mathrm{~A}$ & $-(2)$ \\
\hline & Mediana & $1.219,15 \mathrm{~A}$ & (2) \\
\hline & Basal & $1.198,93 \mathrm{~A}$ & 3. \\
\hline \multirow[t]{2}{*}{ Contagem direta } & Apical & $973,92 \mathrm{~B}$ & (3) \\
\hline & Mediana & $787,38 \mathrm{~B}$ & \\
\hline \multirow{4}{*}{ Sacola de plástico } & Basal & $619,86 \mathrm{C}$ & $320,97 \mathrm{~A}$ \\
\hline & Apical & $184,26 \mathrm{D}$ & \\
\hline & Mediana & $183,89 \mathrm{D}$ & $132,00 \mathrm{~B}$ \\
\hline & Basal & $151.67 \mathrm{D}$ & $112.72 \mathrm{~B}$ \\
\hline
\end{tabular}

(1)Médias seguidas pela mesma letra, na coluna, não diferem entre si pelo teste de Scott-Knott a 5\% de probabilidade. ${ }^{(2)}$ Técnica de amostragem não testada para ninfas. ${ }^{(3)}$ Valor não determinado em virtude da variância relativa ser maior que $25 \%$. gem direta em folha dos terços apical e mediano do espaldeiramento e com o ensacolamento de folha do terço apical, se devem às baixas densidades detectadas por esses sistemas amostrais, já que a variância relativa é inversamente proporcional à densidade (Pedigo, 1988).

Os sistemas com menores tempo e custo unitário na amostragem de adultos de $B$. tabaci foram a bati$\mathrm{da}$, em bandeja, de folha dos terços apical e mediano do espaldeiramento (Tabela 2), ao passo que o sistema com menor tempo e custo unitário na amostragem de ninfas foi a contagem direta em folha do terço basal.

Segundo Kogan \& Herzorg (1980) e Gusmão (2000), quanto maiores os valores de precisão econômica, melhor será o sistema amostral. O sistema mais precioso economicamente na amostragem de adultos foi a batida de folha em bandeja, tanto do terço apical, mediano ou basal do espaldeiramento (Tabela 2), por apresentar variâncias relativas semelhantes aos demais sistemas amostrais. O sistema com maior precisão econômica na amostragem de ninfas foi a contagem direta em folha do terço basal, fato atribuído tanto ao seu baixo custo como à variância relativa.

Os dados dos sistemas selecionados na amostragem de adultos e ninfas pela precisão econômica não se ajustaram às distribuições binomial positiva e Poisson. Entretanto, na maioria das lavouras, estes dados se ajustaram à distribuição binomial negativa (Tabela 3). Esse ajuste indica que os dados amostrais apresentam um padrão de distribuição estatística agregada (Tonhasca et al., 1994; Krebs, 1998).

As curvas de homogeneidade do parâmetro K da distribuição binomial negativa dos sistemas selecionados de amostragem de adultos apresentaram inclinações significativas e interceptos não-significativos (Tabela 4). Portanto, é possível obter-se, para estes sistemas, plano de amostragem envolvendo todas as lavouras, já que essas apresentam um valor comum para o parâmetro $\mathrm{K}$ da distribuição binomial negativa (Bliss \& Owens, 1958). Entretanto, entre estes planos, somente o de amostragem com batida de folha do terço mediano do espaldeiramento em bandeja, a $25 \%$ de precisão, apresentou tempo de amostragem inferior a uma hora $(0,86$ hora $)$ (Tabela 5$)$. 
Este limite é utilizado na seleção de sistemas que irão compor planos de amostragem praticáveis para hortaliças (Gusmão, 2000). Esse critério foi adotado por permitir ao agricultor realizar a amostragem sem comprometer o uso da mão-de-obra necessária às práticas culturais inerentes à atividade hortícola.

A curva de homogeneidade do parâmetro $\mathrm{K}$ da distribuição binomial negativa do sistema selecionado de amostragem de ninfas apresentou inclinação e intercepto significativos (Tabela 3). Portanto, não é possível obter-se, para este sistema, plano de amostragem envolvendo todas as lavouras, já que essas não apresentam um valor comum em relação ao parâmetro $\mathrm{K}$ da distribuição binomial negativa (Bliss \& Owens, 1958). O sistema mais adequado na amostragem de ninfas (contagem direta em folha do terço basal), em todos os níveis de precisão, não possibilitou a geração de plano amostral com número de amostra praticável, visto que os tempos requeridos foram muito superiores ao limite de uma hora (Gusmão, 2000).

Tabela 3. Teste qui-quadrado $\left(\chi^{2}\right)$ entre as frequiências observadas e esperadas pelas distribuições binomial negativa, Poisson e binomial positiva das densidades de adultos de Bemisia tabaci, amostrados por batida, em bandeja, de folha do terço apical (BA), mediano (BM) e basal (BB) do espaldeiramento e das densidades de ninfas, amostradas por contagem direta em folha do terço basal (CDB) do espaldeiramento em pepino.

\begin{tabular}{|c|c|c|c|c|c|c|c|}
\hline \multirow[t]{2}{*}{ Lavoura $^{(1)}$} & \multirow[t]{2}{*}{ Amostragem } & \multicolumn{2}{|c|}{ Binomial negativa } & \multicolumn{2}{|c|}{ Poisson } & \multicolumn{2}{|c|}{ Binomial positiva } \\
\hline & & $\chi^{2}$ & GL & $\chi^{2}$ & GL & $\chi^{2}$ & GL \\
\hline & & \multicolumn{6}{|c|}{ Adultos } \\
\hline 1 & BA & $32,28 *$ & 5 & $256,80^{*}$ & 5 & $5.776,75^{*}$ & 6 \\
\hline 2 & $\mathrm{BA}$ & $6,27^{\mathrm{ns}}$ & 4 & $1.625,21^{*}$ & 7 & $4.545,72^{*}$ & 6 \\
\hline 3 & BA & $82,77^{*}$ & 4 & $804,76^{*}$ & 8 & $117,31^{*}$ & 5 \\
\hline 4 & BA & $18,71^{*}$ & 4 & $140,14^{*}$ & 4 & $4.333,85^{*}$ & 6 \\
\hline 5 & BA & $1,34^{\text {ns }}$ & 2 & $121,16^{*}$ & 2 & $391,62 *$ & 3 \\
\hline 6 & $\mathrm{BA}$ & $0,55^{\mathrm{ns}}$ & 2 & $23,12 *$ & 1 & $417,35^{*}$ & 4 \\
\hline 7 & BA & $22,41^{\text {ns }}$ & 15 & $447,33^{*}$ & 10 & $3.110,82^{*}$ & 2 \\
\hline 8 & BA & $4,12^{\mathrm{ns}}$ & 8 & $262,79 *$ & 7 & $15.601,68^{*}$ & 5 \\
\hline 9 & BA & $4,45^{\mathrm{ns}}$ & 5 & $98,44^{*}$ & 4 & $6.734,01 *$ & 6 \\
\hline 10 & BA & $237,90 *$ & 5 & $516,50^{*}$ & 9 & $9.142,50^{*}$ & 3 \\
\hline 1 & $\mathrm{BM}$ & $6,59^{\text {ns }}$ & 4 & $72,21 *$ & 3 & $4.615,77^{*}$ & 6 \\
\hline 2 & $\mathrm{BM}$ & $2,89^{\mathrm{ns}}$ & 3 & $28,57 *$ & 2 & $2.617,56^{*}$ & 6 \\
\hline 3 & $\mathrm{BM}$ & $10,06^{\mathrm{ns}}$ & 6 & $281,02 *$ & 5 & $1.901,29 *$ & 5 \\
\hline 4 & $\mathrm{BM}$ & $18,99 *$ & 4 & $149,41 *$ & 4 & $4.330,28^{*}$ & 6 \\
\hline 6 & $\mathrm{BM}$ & $0,09^{\mathrm{ns}}$ & 1 & $4,69 *$ & 1 & $409,41^{*}$ & 3 \\
\hline 7 & BM & $136,17 *$ & 9 & $1.120,39^{*}$ & 11 & $34.840,11^{*}$ & 2 \\
\hline 8 & $\mathrm{BM}$ & $3,93^{\text {ns }}$ & 4 & $39,90^{*}$ & 3 & $4.927,52 *$ & 6 \\
\hline 9 & $\mathrm{BM}$ & $13,14^{\text {ns }}$ & 7 & $218,07^{*}$ & 6 & $10.405,94 *$ & 6 \\
\hline 10 & $\mathrm{BM}$ & $13,99^{\text {ns }}$ & 8 & $280,62 *$ & 7 & $6.922,93^{*}$ & 5 \\
\hline 1 & $\mathrm{BB}$ & $9,24^{\mathrm{ns}}$ & 6 & $120,58^{*}$ & 4 & $4.049,37^{*}$ & 6 \\
\hline 2 & $\mathrm{BB}$ & $1,44^{\text {ns }}$ & 3 & $58,79^{*}$ & 2 & $1.619,12^{*}$ & 5 \\
\hline 3 & $\mathrm{BB}$ & $3,04^{\mathrm{ns}}$ & 5 & $185,95^{*}$ & 4 & $4.329,61^{*}$ & 6 \\
\hline 4 & $\mathrm{BB}$ & $5,89^{\mathrm{ns}}$ & 4 & $77,83^{*}$ & 3 & $3.055,50^{*}$ & 6 \\
\hline 6 & BB & $1,82^{\mathrm{ns}}$ & 2 & $21,95^{*}$ & 1 & $540,26^{*}$ & 4 \\
\hline 7 & $\mathrm{BB}$ & $17,61^{\mathrm{ns}}$ & 10 & $749,51 *$ & 12 & $2.717,69^{*}$ & 2 \\
\hline 8 & BB & $4,83^{\text {ns }}$ & 5 & $141,38^{*}$ & 4 & $2.513,63^{*}$ & 5 \\
\hline 9 & BB & $11,41^{\mathrm{ns}}$ & 7 & $163,72 *$ & 6 & $6.718,79^{*}$ & 5 \\
\hline 10 & $\mathrm{BB}$ & $9.22^{\mathrm{ns}}$ & 6 & $144.42 *$ & 5 & $6.152 .00 *$ & 6 \\
\hline \multicolumn{8}{|c|}{ Ninfas } \\
\hline 1 & $\mathrm{CDB}$ & $8,82^{\mathrm{ns}}$ & 4 & $1.905,63^{*}$ & 12 & $2.924,32 *$ & 5 \\
\hline 2 & $\mathrm{CDB}$ & $3,80_{\mathrm{ns}}^{\mathrm{ns}}$ & 3 & $418,86^{*}$ & 4 & $2.789,75^{*}$ & 6 \\
\hline 3 & CDB & $9,20^{\text {ns }}$ & 8 & $1.352,98^{*}$ & 9 & $2.845,24 *$ & 5 \\
\hline 4 & CDB & $13,10^{\text {ns }}$ & 7 & $1.271,51^{*}$ & 7 & $3.768,58^{*}$ & 6 \\
\hline 5 & CDB & $0,28^{\mathrm{ns}}$ & 1 & $38,19^{*}$ & 1 & $48,18^{*}$ & 1 \\
\hline 6 & CDB & $8,08^{\text {ns }}$ & 4 & $1.273,35^{*}$ & 8 & $4.281,88^{*}$ & 6 \\
\hline 7 & $\mathrm{CDB}$ & $253,48 *$ & 2 & $357,62 *$ & 19 & $26.991,83^{*}$ & 3 \\
\hline 8 & CDB & $54,33^{*}$ & 4 & $1.024,72 *$ & 15 & $5.835,38^{*}$ & 6 \\
\hline 9 & $\mathrm{CDB}$ & $3,59^{\mathrm{ns}}$ & 4 & $1.947,78^{*}$ & 8 & $5.539,93^{*}$ & 6 \\
\hline 10 & $\mathrm{CDB}$ & $56,65^{*}$ & 5 & $1.606,58^{*}$ & 14 & $3.370,10^{*}$ & 5 \\
\hline
\end{tabular}

${ }^{(1)} \mathrm{Na}$ lavoura 5 não detectaram-se adultos de $B$. tabaci pela batida, em bandeja, de folha do terço mediano e basal do espaldeiramento. ${ }^{\text {ns Não-significativo }}$ *Significativo a $5 \%$ de probabilidade. 
Tabela 4. Estimativa e teste de homogeneidade em relação ao parâmetro $\mathrm{K}$ da distribuição binomial negativa para os dados de densidades de adultos e de ninfas de Bemisia tabaci (Hemiptera: Aleyrodidae), amostrados pelos sistemas selecionados com base na precisão econômica.

\begin{tabular}{|c|c|c|c|c|c|}
\hline Técnica & $\begin{array}{c}\text { Parte do } \\
\text { esnaldeiramento }\end{array}$ & $\begin{array}{l}\text { Fase do } \\
\text { inseto }\end{array}$ & $\mathrm{K}^{\text {comum }}$ & Inclinacão & Inters \\
\hline Batida em bandeja & Apical & Adulta & 0,27 & $18,89^{*}$ & $1,40^{\mathrm{ns}}$ \\
\hline Batida em bandeja & Mediana & Adulta & 0,54 & $37,04 *$ & $0,43^{\mathrm{ns}}$ \\
\hline Batida em bandeja & Basal & Adulta & 0,62 & $42,30^{*}$ & $2,38^{\mathrm{ns}}$ \\
\hline Contagem direta & Basal & Ninfal & 0,21 & $53,59^{*}$ & $37,30 *$ \\
\hline
\end{tabular}

Tabela 5. Número total de amostras, tempo e custo da amostragem de adultos Bemisia tabaci (Hemiptera: Aleyrodidae) por batida em bandeja de folha do terço apical, mediano e basal do espaldeiramento do pepino, em razão do nível de precisão do plano de amostragem. Tocantins, MG, 2001.

\begin{tabular}{rccc}
\hline Precisão (\%) & Número & Tempo (horas) & Custo (R $\$$ ) \\
\hline 5 & & Apical & \\
5 & 7.382 & 34,43 & 55,37 \\
10 & 1.846 & 8,61 & 13,84 \\
15 & 820 & 3,83 & 6,15 \\
20 & 461 & 2,15 & 3,46 \\
25 & 295 & 1,38 & 2,21 \\
\hline & \multicolumn{4}{c}{ Mediana } \\
5 & 4.897 & 21,40 \\
10 & 1.224 & 5,35 & 34,28 \\
15 & 544 & 2,38 & 8,57 \\
20 & 306 & 1,34 & 3,81 \\
25 & 196 & 0,86 & 2,14 \\
& & Basal & 1,37 \\
5 & 13.051 & 102,71 & 164,45 \\
10 & 1.335 & 10,51 & 16,82 \\
15 & 522 & 4,11 & 6,58 \\
20 & 283 & 2,22 & 3,56 \\
25 & 178 & 1,40 & 2,24 \\
\hline
\end{tabular}

\section{Conclusões}

1. Em nenhum sistema amostral para ninfas de B. tabaci o tempo de amostragem é praticável.

2. A amostragem da mosca-branca $B$. tabaci no pepino deve ser feita amostrando-se seus adultos por batida de 196 folhas do terço mediano do espaldeiramento em bandeja de plástico branca.

\section{Agradecimentos}

Ao Conselho Nacional de Desenvolvimento Científico e Tecnológico (CNPq), à Coordenação de Aperfeiçoamento de Pessoal de Nível Superior (Capes) e à Fundação de Amparo à Pesquisa do Estado de Minas Geras (Fapemig) pelo auxílio financeiro e bolsas concedidas.

\section{Referências}

BARBOSA, J. C.; PERECIN, D. Modelos probabilísticos para distribuições de Spodoptera frugiperda (J.E. Smith, 1997), na cultura do milho. Científica, São Paulo, v. 10, n. 2, p. 181-191, dez. 1982.

BLISS, C. I.; OWENS, A. R. G. Negative binomial distributions with a common K. Biometrika, London, v. 45 , n. $1 / 2$, p. $37-58$, June 1958

BROWN, J. K.; FROHLICH, D. R.; ROSELL, R. C. The sweet potato or silverleaf whiteflies: biotypes of Bemisia tabaci or a species complex? Annual Review of Entomology, Palo Alto, v. 40, p. 511-534, Dec. 1995.

ELLSWORTH, P. C.; CARRILLO, J. L. M. IPM for Bemisia tabaci: a case study from North America. Crop Protection, Oxford, v. 20, n. 9, p. 853-869, Nov. 2001.

FILGUEIRA, F. A. R. Novo manual de olericultura: agrotecnologia moderna e comercialização de hortaliças. Viçosa, MG: UFV, 2000. 402 p.

GUSMÃO, M. R. Avaliação de vetores de viroses, predadores e parasitóides e plano de amostragem para mosca-branca do tomateiro. 2000. 42 f. Dissertação (Mestrado em Entomologia) - Universidade Federal de Viçosa, Viçosa, MG, 2000.

KOGAN, M.; HERZOG, D. C. Sampling methods in soybean entomology. New York: Springer, 1980. 587 p.

KREBS, J. C. Ecological methodology. Melon Park: University of British Columbia, 1998. 620 p.

LOURENÇÃO, A. L.; NAGAI, H. Surtos populacionais de Bemisia tabaci no Estado de São Paulo. Bragantia, Campinas, v. 53, n. 1, p. 53-59, out. 1994.

MIDGARDEN, D. G.; YOUNGMAN, R. R.; FLEISCHER, S. J. Spatial analysis of counts of western corn rootworm (Coleoptera: Chrysomelidae) adults on yellow sticky traps in corn: geostatistics and dispersion indices. Environmental Entomology, Lanham, v. 22, n. 5, p. 1124-1133, Oct. 1993.

MIRANDA, M. M. M.; PICANÇO, M. C.; MATIOLI, A. L.; PALLINI FILHO, A. Distribuição na planta e controle biológico natural de pulgões (Homoptera: Aphididae) em tomateiro. Revista Brasileira de Entomologia Curitiba, v. 42, n. 1, p. 13-16, jun. 1998.

NARANJO, S. E. Conservation and evaluation of natural enemies in IPM systems for Bemisia tabaci. Crop Protection, Oxford, v. 20, n. 9, p. 835-852, Nov. 2001. 
NARANJO, S. E.; FLINT, H. M. Spatial distribution of preimaginal Bemisia tabaci (Homoptera: Aleyrodidae) in cotton and development of fixed-precision sequential sampling plans. Environmental Entomology, Lanham, v. 23, n. 2, p. 254-266, Apr. 1994.

NAULT, B. A.; KENNEDY, G. G. Sequential sampling plans for use in timing insecticide applications for control of European corn borer (Lepidoptera: Pyralidae) in potato. Journal of Economic Entomology, Lanham, v. 89, n. 6, p. 1468-1476, Dec. 1996.

PAULA, S. V.; PICANÇO, M. C.; VILELA, E. F.; FONTES, P.C. R. Incidência de insetos vetores de fitovírus em tomateiro (Lycopersicon esculentum Mill.) circundado por faixas de culturas. Revista Brasileira de Entomologia, Curitiba, v. 41, n. 3, p. 555-558, out. 1997.

PEDIGO, L. P. Entomology and pest management. New York: Macmillan, 1988. 646 p.

PEDIGO, L. P.; BUTIN, G. D.; BECHINSKI, E. J. Flushing technique and sequential-count plan for green cloverworm (Lepidoptera: Noctuidae) moths in soybeans. Environmental Entomology, Lanham, v. 11, n. 6, p. 1223-1228, Dec. 1982.

PICANÇO, M. C.; GUEDES, R. N. C. Manejo integrado de pragas no Brasil: situação atual, problemas e perspectivas. Ação Ambiental, Viçosa, MG, v. 2, n. 4, p. 23-26, jan. 1999.

PICANÇO, M. C.; MARQUINI, F.; GALVAN, T. L. Manejo de pragas em cultivos irrigados sob pivô central.
In: ZAMBOLIM, L. (Ed.). Manejo integrado fitossanidade: cultivo protegido, pivô central e plantio direto. Visconde do Rio Branco: Suprema, 2001. p. $427-$ 480.

SCHUSTER, D. J. Intraplant distribution of immature life stages of Bemisia argentifolii (Homoptera: Aleyrodidae) on tomato. Environmental Entomology, Lanham, v. 27, n. 1, p. 1-9, Feb. 1998.

SilVA, A. C.; AGOSTINI, I.; MUlleR, J. J. V.; VIZZOTO, V. J. Efeito de densidades populacionais sobre a produtividade de pepino para conserva. Horticultura Brasileira, Brasília, v. 10, n. 1, p. 28-29, maio 1992.

SOUTHWOOD, T. R. E. Ecological methods. London: Chapman and Hall, 1978. 524 p.

TONHASCA, A.; PALUMBO, J. C.; BYRNE, D. N. Distribution patterns of Bemisia tabaci (Homoptera: Aleyrodidae) in cantaloupe fields in Arizona. Environmental Entomology, Lanham, v. 23, n. 4, p. 949954, Aug. 1994.

WAQUIL, J. M. Amostragem e abundância de danos de Dalbulus maidis (Delong \& Wolcott) (Homoptera: Cicadellidae) em plântulas de milho. Anais da Sociedade Entomológica do Brasil, Londrina, v. 26, n. 1, p. 27-33, abr. 1997.

YOUNG, L. J.; YOUNG, J. H. Statistical ecology: a population perspective. Boston: Kluwer Academic, 1998. 565 p. 